\title{
Recomendação de Jogos na Aprendizagem da Matemática baseado na Análise Diagnóstica e Teoria de Resposta ao Item
}

\author{
Walkir, A.T. Brito ${ }^{1}$, Claudia L.R. Motta ${ }^{2}$ \\ ${ }^{1}$ Programa de Pós-Graduação em Informática - PPGI/UFRJ \\ Universidade Federal do Rio de Janeiro - Rio de Janeiro, RJ - Brazil \\ ${ }^{2}$ Programa de Pós-Graduação em Informática - PPGI \\ Instituto Tércio Pacitti de Aplicações e Pesquisa Computacionais - NCE/UFRJ \\ Universidade Federal do Rio de Janeiro - Rio de Janeiro, RJ - Brazil \\ walkir.brito@gmail.com, claudiamence.ufrj.br
}

\begin{abstract}
Considering the necessity to allow for a new approach in teaching mathematics in high school that encourages creativity, involvement and conscious learning, this paper presents the methodological strategy of using the recommendation of games in mathematics teaching based on diagnostic analysis method and using educational evaluation as the item response theory (IRT) to validate educational games recommended on application of the system along the classes of 1st year of high school.
\end{abstract}

Resumo. A partir da necessidade de permitir um novo enfoque no ensino da matemática no ensino médio que estimule a criatividade, envolvimento e um aprendizado consciente, o presente trabalho apresenta a estratégia metodológica do uso da recomendação de Jogos no ensino da Matemática baseado no método da análise diagnóstica e utilizando como avaliação educacional a teoria de resposta ao item (TRI), para validar os jogos educacionais recomendados na aplicação do sistema junto a turmas de $1^{\circ}$ ano do ensino médio.

\section{Introdução}

Os jogos constituem um modo interessante de proposição de problemas devido a sua atratividade para crianças e jovens além de favorecerem o exercício da criatividade na elaboração de estratégias de resolução. Podem ainda simular situações-problema que demandam soluções rápidas, estimulando a superação de dificuldades no aprendizado da disciplina e uma atitude positiva perante os erros e quando praticados em grupos, estimular o debate, a argumentação e organização do pensamento, pois jogar implica não somente no aspecto lúdico: a competição entre os alunos pode gerar e desenvolver a atitude de cooperação e trabalho em equipe, garantir uma maior atenção dos alunos durante a aula e o estabelecimento de uma ligação entre as capacidades matemáticas desenvolvidas nos jogos e a realidade que circunda o aluno, estimulando a resolução de problemas ou desafios propostos. Segundo o Ministério da Educação e a Secretaria de Educação Fundamental MEC/SEF (PCN 1998), o professor pode valer-se da história da Matemática, de tecnologias de comunicação e de jogos como recursos que podem fornecer o contexto dos problemas, como também os instrumentos para a construção das 
estratégias de resolução.

Sistemas de recomendação neste trabalho são filtros de informações para apresentar jogos educacionais necessários e interessantes aos alunos, articulando os resultados da aplicação da avaliação diagnóstica aos assuntos em que os alunos apresentam deficiências (Motta, 2011). O resultado da análise diagnóstica é a entrada de dados necessária para o funcionamento das recomendações dos jogos na aprendizagem da matemática que serão geridas pelo sistema. Segundo Casagrande (2014), diversos trabalhos demonstram que sistemas de recomendação oferecem vantagens no contexto educacional. Estes trabalhos propõem a recomendação de conteúdos educacionais descritos por metadados, que visam capturar as informações do usuário implicitamente. No entanto, diferentemente do modelo apresentado neste artigo, são em sua maioria modelos teóricos, sem formalização do procedimento realizado para as recomendações. Temos como exemplo deste tipo de modelo teórico o sistema de recomendação criado por Primo et al. (2013). O modelo proposto por nosso trabalho faz uso de um sistema híbrido de recomendações de jogos educacionais, empregando filtragens colaborativas e habilidades e competências. Este procedimento foi aplicado no ponto inicial da pesquisa e foi descrito na dissertação (Brito, 2014).

Através da utilização de recomendação aliada a jogos educacionais como ferramenta de apoio ao ensino da Matemática este trabalho busca a formação de um ambiente de aprendizagem mais adequado à natureza da matéria e das necessidades apresentadas pelos alunos em seu contexto de aprendizagem. Consiste na busca por essas estratégias de identificação, recomendação, avaliação e medição o problema levantado por esta pesquisa, sem deixar de lado uma reflexão sobre a qualidade da educação básica e como ela pode ser alcançada através de novos enfoques e ferramentas mais coadunadas com os desafios para o ensino e aprendizagem em nossa época. Procura-se oferecer uma abordagem mais amigável, instigante e atraente, tornando a matéria mais interessante, de forma a facilitar o seu aprendizado.

O presente artigo apresenta resultados parciais de uma pesquisa mais ampla que tem como principal objetivo fazer uma reflexão sobre o uso dos jogos no ensino da Matemática, para tornar mais atraente e contextualizado esse processo aos jovens, implicando num melhor desenvolvimento de competências e habilidades e a relação destes alunos com a disciplina, bem como a escolha por parte destes do futuro exercício de profissões diretamente ligadas à utilização da Matemática.

\section{Contextualização da Pesquisa}

Utilizamos a plataforma SESI Matemática desenvolvida pela Federação das Indústrias do Estado do Rio de Janeiro (FIRJAN), e o Serviço Social da Indústria (SESI RJ), que visa contribuir para a melhoria e a qualidade da aprendizagem da Matemática para estudantes do Ensino Médio. A Plataforma SESI Matemática é composta por 79 jogos e desafios que perfazem uma combinação de até 5.000 desafios que contemplam todo o currículo mínimo do ensino fundamental e médio (Programa Sesi Matemática, 2012). Esta ferramenta torna possível que professores e alunos identifiquem os conhecimentos matemáticos por conteúdos (aritmético, geométrico, métrico, algébrico, estatístico, combinatório e probabilístico) ou pela possibilidade de sequenciar os conteúdos das conexões que se estabelecem nos conhecimentos já construídos pelos alunos, não se restringindo à ideia tradicional de pré-requisito ou de uma sucessão de tópicos 
estabelecidos a priori (por ano letivo e ou conteúdo que deve ser visto naquele ano), possibilitando desenvolver esses conhecimentos em diferentes níveis de interatividade.

Esta proposta de trabalho utilizou quatro eixos principais: o ensino da Matemática, cuja amostra considera alunos do $1^{\circ}$ ano do ensino médio, avaliação diagnóstica de habilidades e competências de acordo com a elaboração do Parâmetro Curricular Nacional para o ensino de Matemática do Ministério da Educação (PCN, MEC), a utilização de recomendações que selecionou os jogos educacionais de acordo com as habilidades e competências de cada aluno e a TRI como avaliação educacional dos resultados obtidos.

\section{Metodologia}

Trabalhamos com um modelo de recomendação para filtrar informações relevantes para o estudante e a partir destas selecionar jogos educacionais mais apropriados de acordo com as competências a serem desenvolvidas, por tema ou conteúdo, no ensino da Matemática, respeitando a Matriz de Referência proposta pelo MEC. Este trabalho utilizou o modelo proposto na dissertação (Brito, 2014) e por meio de dois estudos de casos.

O primeiro estudo, em uma escola Sesi, para realizar o estudo do pré teste, a aplicação e teste da análise diagnóstica, a calibração do grau de facilidade dos itens utilizando a teoria de resposta ao item (TRI), a criação da régua dos resultados para análise dos resultados aplicados na análise diagnóstica, pré e pós, na utilização dos jogos recomendados no ensino da matemática para cada estudante, levando em consideração o resultado da análise diagnóstica e as habilidades e competências de cada participante. Serviu também para a correção das questões que apresentaram problemas de entendimentos e principalmente para testar a entrada de dados das recomendações dos jogos, e seu cadastramento na plataforma para a aplicação real do estudo.

O segundo estudo, foi realizado em duas escolas da rede VespeR, no estado de São Paulo. Para aplicar o modelo realizaram-se todas as etapas metodológicas apresentadas no primeiro estudo, além de testarmos as recomendações dos jogos educacionais, as habilidades e competências utilizadas pelos participantes na plataforma, aplicando a análise diagnóstica Pré e Pós, e verificando os estímulos recomendados através da TRI, isto é, após a utilização dos jogos recomendados no ensino da matemática, verificou se houve alguma diferença entre os participantes do estudo.

\section{Teoria de Resposta ao Item (TRI)}

Atualmente, em várias áreas do conhecimento, particularmente em avaliação educacional, vem crescendo o interesse na aplicação de técnicas derivadas da TRI que propõem modelos observando as características do individuo que não podem ser verificadas diretamente. Um dos diferenciais mais importantes refere-se ao processo de identificação e da relação entre as propriedades dos itens e das pessoas, tendo como pontos de partida modelos probabilísticos. Essa característica de acordo com Nunes e Primi [9], é que proporciona ao modelo o termo 'teoria', uma vez que, diante de dados empíricos, que são as respostas dadas por um grupo de pessoas a um conjunto de itens, é construído um modelo que tenta explica a relação ente eles. 
O principal objetivo da TRI é a construção de escalas para traços latentes através da análise das respostas a itens que podem refletir tanto aspectos de personalidade ou atitude, quanto níveis de desempenho ou habilidade. Em todos os modelos de TRI a hipótese básica é a de que as respostas aos itens contêm informação sobre o traço latente a ser medido. A TRI é um conjunto de modelos matemáticos que procuram representar a probabilidade de um indivíduo dar certa resposta a um item como função dos parâmetros do item e da habilidade (ou habilidades) do respondente. Essa relação é sempre expressa de tal forma que quanto maior a habilidade, maior a probabilidade de acerto no item.

O modelo proposto baseia-se no fato de que o indivíduo com maior habilidade (traço latente $\theta$ ) possui maior probabilidade de acertar o item e que esta relação não é linear. Para a análise dos dados, foram consideradas as respostas dos participantes a uma das cinco alternativas de cada questão na análise diagnóstica, usamos uma escala de -4 a 4 para representação e calibramos (processo de estimação dos parâmetros dos itens) utilizando o software: R versão 3.0.2 e o software SPSS. Indicadas as respostas corretas, os dados foram transformados em itens do tipo: certo ou errado (itens dicotômicos) e analisados. Os resultados foram equalizados e apresentados no formato de régua, isto é, a habilidade das pessoas ( $\theta$ ), é colocada na mesma escala da dificuldade das questões (equalização). Assim ao analisarmos a mesma métrica para a habilidade dos testados e para a dificuldade das questões, podemos comparar e visualizar graficamente as probabilidades de acerto da questão. Vejamos como exemplo, na Figura 1. O estudante "Joãozinho" e a imaginária questão 7 na escala de habilidade $(\theta)$ :como o $\theta$ de Joãozinho $(2,0)$ é maior que o $\theta$ da Questão $7(1,0)$ a probabilidade de que Joãozinho acerte a questão 7 é maior que $50 \%$.

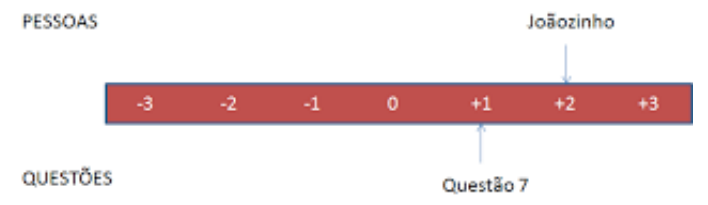

Figura 1. Régua Equalizada Probabilidade Maior de Acerto

Para a questão 8 fictícia abaixo, o $\theta$ é de 3,0, como é possível observar na Figura 2. Nesse caso, o $\theta$ de Joãozinho $(2,0)$ é menor que o $\theta$ da Questão $8(3,0)$, a probabilidade de que ele acerte essa questão é menor que $50 \%$ e quando for igual o $\theta$ da questão e do participante a probabilidade seria igual a $50 \%$.

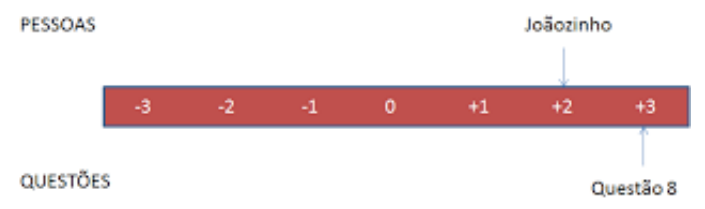

Figura 2. Régua Equalizada Probabilidade Menor de Acerto

Experiências relatadas em trabalhos acadêmicos e nos meios de comunicação têm apontado resultados positivos no emprego deste método. Portanto, pressupomos que aliar ferramentas de recomendação à aplicação de jogos para sanar dificuldades e estimular habilidades constitui-se uma maneira extremamente positiva no alcance de 
resultados que beneficiem a relação de ensino aprendizagem da Matemática, tanto para alunos quanto professores.

\section{Avaliação Diagnóstica}

As questões apresentadas na avaliação diagnóstica foram elaboradas por professores indicados pelo Instituto Nacional de Matemática Pura e Aplicada (IMPA). A abordagem foi baseada nas habilidades e competências que estão nos currículos das unidades da Federação e nos Parâmetros Curriculares Nacionais. E foram aplicadas ao $1^{\circ}$ ano do Ensino Médio. A avaliação é composta por 15 questões concernentes a toda a estrutura de descritores que compõem a matriz de habilidades e competências e tem por objetivo identificar dificuldades de aprendizagem do educando e suas possíveis causas objetivando orientar futuras ações de solução a esses problemas. Após a aplicação da prova, os dados são processados e as respostas de cada questão analisadas gerando as recomendações necessárias dos jogos que cada estudante deverá utilizar para a seleção dos conteúdos que serão trabalhadas pelo professor. Todas as recomendações são agendadas e inseridas dentro da Plataforma, onde cada estudante tem acesso às recomendações dos jogos educacionais. A avaliação diagnóstica foi separada em cinco grupos, segundo as habilidades e competências (Tabela 1). Optamos por trabalhar com os Grupos: 1 e 5 nos resultados desse trabalho por representarem um alto percentual do índice significativo na amostra.

Tabela 1. Avaliação Diagnóstica por Grupos, Habilidades e Competências.

\begin{tabular}{|c|c|c|c|c|c|c|}
\hline Grupos & \multicolumn{6}{|c|}{ Habilidades e Competências } \\
\hline $\begin{array}{l}\text { Grupo 1: Números Reais } \\
\text { œQuestões: 1,3, } 5 \text { e } 6 \text {. }\end{array}$ & $\begin{array}{l}\text { Resolver problemas } \\
\text { utilizando as operações } \\
\text { fundamentais no } \\
\text { conjunto dos números } \\
\text { reais. }\end{array}$ & \begin{tabular}{|l|} 
Reconhecer e \\
diferenciar \\
números decimais \\
finitos ou infinitos, \\
periódicos e não \\
periódicos.
\end{tabular} & $\begin{array}{l}\text { Ordenar e comparar } \\
\text { números reais }\end{array}$ & $\begin{array}{l}\text { Identificar a } \\
\text { localização de } \\
\text { números reais na reta } \\
\text { numérica. }\end{array}$ & & \\
\hline $\begin{array}{l}\text { Grupo 2: Conjuntos } \propto \\
\text { Questões: 2, 4, } 12 \text { e } 14 .\end{array}$ & $\begin{array}{l}\text { Compreender a noção de } \\
\text { conjunto. }\end{array}$ & \begin{tabular}{|l} 
Utilizar a \\
simbologia \\
matemática para \\
compreender \\
proposições e \\
enunciados.
\end{tabular} & $\begin{array}{l}\text { Resolver problemas } \\
\text { significativos } \\
\text { envolvendo operações } \\
\text { com conjuntos. }\end{array}$ & $\begin{array}{l}\text { Reconhecer e } \\
\text { diferenciar os } \\
\text { conjuntos numéricos. }\end{array}$ & $\begin{array}{l}\text { Identificar a } \\
\text { localização de } \\
\text { números reais na reta } \\
\text { numérica. }\end{array}$ & $\begin{array}{l}\text { Utilizar a representação } \\
\text { de números reais na reta } \\
\text { para resolver problemas } \\
\text { e representar } \\
\text { subconjuntos dos } \\
\text { números reais. }\end{array}$ \\
\hline $\begin{array}{l}\text { Grupo 3: Estudo de } \\
\text { Funções œQuestões: } 7 \text { e } \\
\text { 9. }\end{array}$ & $\begin{array}{l}\text { Compreender o conceito } \\
\text { de função através da } \\
\text { dependência entre } \\
\text { variáveis. }\end{array}$ & \begin{tabular}{|l} 
Identificar a \\
expressão algébrica \\
que expressa uma \\
regularidade ou \\
padrão.
\end{tabular} & $\begin{array}{l}\text { Representar pares } \\
\text { ordenados no plano } \\
\text { cartesiano. }\end{array}$ & $\begin{array}{l}\text { Construir gráficos de } \\
\text { funções utilizando } \\
\text { tabelas de pares } \\
\text { ordenados. }\end{array}$ & $\begin{array}{l}\text { Analisar gráficos de } \\
\text { funções (crescimento, } \\
\text { decrescimento, zeros, } \\
\text { variação do sinal). }\end{array}$ & \\
\hline $\begin{array}{l}\text { Grupo 5: Razões } \\
\text { Trigonométricas no } \\
\text { triângulo retângulo œ } \\
\text { Questão: 11. }\end{array}$ & $\begin{array}{l}\text { Utilizar as razões } \\
\text { trigonométricas para } \\
\text { calcular o valor do seno, } \\
\text { cosseno e tangente, dos } \\
\text { ângulos de } 30^{\circ}, 45^{\circ} \text { e } \\
60^{\circ} \text {. }\end{array}$ & \begin{tabular}{|l} 
Resolver \\
problemas do \\
cotidiano \\
envolvendo as \\
razões \\
trigonométricas.
\end{tabular} & $\begin{array}{l}\text { Utilizar os teoremas } \\
\text { do seno e do cosseno } \\
\text { para resolver } \\
\text { problemas } \\
\text { significativos. }\end{array}$ & & & \\
\hline
\end{tabular}

\section{Etapas Utilizadas na Aplicação do Estudo}

Com o propósito de aprofundar os resultados alcançados nesse estudo os alunos participantes foram convidados a responder perguntas sobre a satisfação da utilização e 


\section{Resultados}

Fazendo uma análise comparativa entre os resultados encontrados na avaliação diagnostica Pré versus Pós, as utilizações dos jogos revelaram alguns aspectos importantes, tais como: houve um aumento na média geral dos níveis dos alunos, quando comparado à utilização dos jogos na aplicação da régua, podemos considerar esse crescimento como sendo o aumento da habilidade $(\theta)$ tornando o participante com maior habilidade para o item testado.

Obtivemos como resultado principal uma melhoria de $74 \%$ (Figura 4), após a utilização dos jogos, analisando a posição inicial do participante versus a posição final, houve uma movimentação de crescimento de todos os participantes na Régua de Resultados utilizada no estudo. Esse movimento de crescimento entre a relação do número de participantes versus escala de classificação de pontos ocorreu em todas as escalas da régua de resultados. Já para os participantes que obtiveram resultados acima do esperado houve um crescimento significativo, visto que seis participantes $(11 \%)$ apresentam um resultado acima do esperado e ocorreu um crescimento para vinte e nove participantes $(55 \%)$, mostrando um crescimento de desempenho significativo de uma avaliação para a outra.

Após a análise dos resultados do estudo conclui-se que Jogos recomendados e oferecidos aos alunos oferecem um imprescindível suporte pedagógico e metodológico às relações de ensino e aprendizagem na Matemática, visto que representam um diferencial de inovação ao ensino dos conteúdos básicos necessários ao desenvolvimento do raciocínio lógico matemático, bem como das habilidades e competências previstas nos parâmetros curriculares nacionais do ensino médio. Concluímos ainda que os alunos que apresentam maior pré-disposição na aceitação dessa nova metodologia de ensino, através da utilização de jogos na resolução de situações problemas, apresentam também melhores resultados sendo capazes de validar estratégias e resultados e desenvolver novas formas de raciocínio e processos que implicam em um aumento de seu raciocínio lógico matemático e de seu desempenho para dedução, indução, analogia e estimativas.

A utilização de recomendação, que trabalhe precipuamente com a análise diagnóstica e ofereça jogos específicos que considerem os interesses, particularidades, necessidades e dificuldades pedagógicas e metodológicas de cada aluno, com objetivo de desenvolvimento e construção cognitivos se configura como um eficiente instrumento de ensino-aprendizagem da Matemática. Após a análise de resultados do estudo verificamos que os jogos são instrumentos pedagógicos e metodológicos efetivos na produção e apropriação de conhecimentos, no desenvolvimento de habilidades e competências e na utilização de raciocínio matemático lógico, visto que os resultados obtidos mostram que ocorreu um ganho significativo na aprendizagem da matemática para os alunos participantes que utilizaram os jogos recomendados através da Plataforma. A utilização de uma avaliação diagnóstica fidedigna, aliada a recomendação de jogos no ensino da matemática propicia uma visão holística do aluno e suas reais necessidades. Utilizou-se como diferencial nesse estudo a TRI, que se propõem a avaliar o desenvolvimento dos alunos, permitiu ainda que os desempenhos dos participantes pudessem ser comparados e colocados em uma escala única de 
conhecimento. Dessa forma a recomendação de jogos específicos torna-se eficaz, eficiente e efetiva, visto que de acordo com a TRI quanto maior a habilidade maior a probabilidade de acerto no item.

Conclui-se que foi de extrema validade o primeiro estudo (Brito, 2014), quanto à aplicação e utilização da teoria de resposta ao item, assim como a calibração do grau de dificuldade ou escala de facilidade do item, permitindo sua utilização nesse estudo. Além disso, através do teste das hipóteses levantadas, constatou-se que a utilização de recomendação aliado a avaliação diagnóstica previa que indicasse jogos, levando em consideração os interesses, especificidades, e as necessidades pedagógicas e metodológicas de cada aluno, constitui-se um eficiente instrumento no ensino da Matemática e é uma importante contribuição desse estudo.

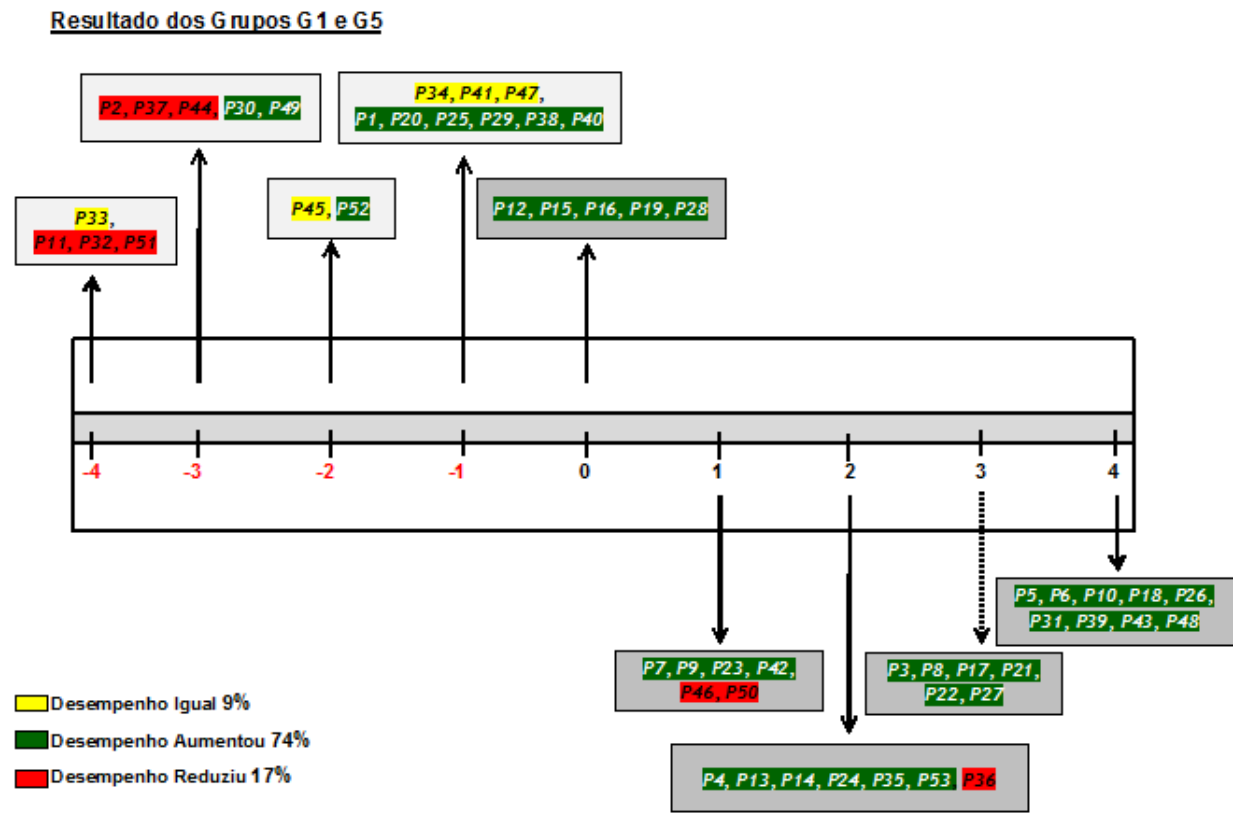

Figura 4. Régua dos resultados

\section{Considerações Finais}

Este artigo apresentou recomendação de Jogos educacionais no ensino aprendizagem da Matemática, baseado a partir da análise diagnóstica e utilizando como avaliação educacional a TRI, para validar os jogos recomendados na aplicação do sistema junto a turmas de primeiro ano do ensino médio. Buscando despertar, desta forma, um dos objetivos primordiais no processo de ensino e aprendizagem: questionar a realidade, formulando problemas e tratando de resolvê-los, utilizando para isso o pensamento lógico, a criatividade, a intuição, a capacidade de análise crítica, selecionando procedimentos e verificando sua adequação. Gerar a partir da realização deste estudo um novo enfoque do ensino que não se limite à exploração estritamente gradual dos conteúdos e temas da Matemática. Mas sim a resolução de problemas reais, procurando criar um novo caminho no ensino da disciplina tão importante na construção do saber e contribuindo para acabar com os mitos e medos sobre o tema. Como trabalhos futuros pretende-se incluir a pesquisa aplicação sistemática em uma nova escola, pelo período de um semestre letivo, testando o aumento das habilidades 
cognitivas no desenvolvimento de habilidades e competências, além da observação da formação de duplas e grupos de participantes, no intuito de desenvolver a capacidade de trabalho em conjunto, promovendo a sociabilidade e desenvolvimento do espírito de liderança.

\section{Referências}

Ministério da Educação. Parâmetros Curriculares Nacionais, ( $1^{\mathrm{a}}$ a $4^{\mathrm{a}}$ Série) PCN, (1998), Secretaria de Educação fundamental - Matemática, Ministério de Educação e Desporto, MEC/SEF.

CASAGRANDE, M.F.R et al. Técnicas de recomendação baseada em metadados para repositórios digitais voltados ao ensino. Anais do Simpósio Brasileiro de Informática na Educação, 2013. Disponível em: www.br-ie.org/pub/index.php/sie. Acessado em: 11 Set. 2014.

BRITO, W.A.T. Modelos de Recomendação de Jogos educacionais Baseado em Seleção de Conteúdo no ensino da Matemática. Dissertação de Mestrado em Informática, PPGI, Universidade Federal do Rio de Janeiro, 2014.

FERRAREZI, L. A. A importância do jogo no resgate do ensino de geometria, In: Anais do VIII ENEM - UFPE, Recife, 2004, p.3.

FLEMMING, D.M, COLLAÇO DE MELLO, A.C. Criatividade Jogos Didáticos, São José: Saint-Germain, 2003.

PASQUALI, L., \& Primi, R. (2007). Fundamentos da Teoria da Resposta ao Item TRI. Em L. Pasquali (Org.). Teoria de resposta ao Item: TRI, (11-28). Brasília, Distrito Federal: Editora UNB.

Plataforma Sesi Matemática. Coordenação de Sistema Firjan, 2012. Disponível em: http://www.mangahigh.com. Acesso em: 11 Set. 2014.

PRIMO, T.T. et al. Rumo ao uso de metadados educacionais em sistema de recomendação. Simpósio Brasileiro de Informática na Educação, 2013, p. 4-8.

Programa Sesi Matemática. Desenvolvido pelo Sistema Firjan e Sesi Rio, 2012. Disponível em: http://www.firjan.org.br/sesimatematica/. Acesso em: 11 Set. 2014.

MOTTA, C.L.R. et al. Sistemas de recomendação. In: Sistemas Colaborativos. PIMENTEL e FUKS (Org). Rio de Janeiro: Elsevier, 2011.

NUNES, C. H. S. S., \& Primi, R. (2009). Teoria de Resposta ao Item: Conceitos e aplicações na Psicologia e na Educação. Em: C. S. Hutz (Org.). Avanços e polêmicas em avaliação psicológica, (pp. 25-69). São Paulo: Casa do Psicólogo.[14]

RICCI, F., ROKACH, L., SHAPIRA, B., KANTOR, P.B. Recommender Systems Handbook. Springer, 2011. 